\title{
MODELOS LOGÍSTICOS APLICADOS EN LA DISTRIBUCIÓN URBANA DE MERCANCÍAS
}

\author{
MARTÍn DARÍo ARANGo SERnA ${ }^{1}$ \\ Cristian Giovanny Gómez Marín ${ }^{2}$ \\ ConRado Auguto SERna URÁN ${ }^{3}$
}

\section{RESUMEN}

Este artículo presenta una clasificación de los modelos logísticos que se han desarrollado para dar solución a los problemas de integración, coordinación, sostenibilidad y movilidad que surgen en la distribución urbana de mercancías (DUM). La búsqueda de la mejora y optimización de los procesos en los sistemas de distribución de bienes y servicios en zonas urbanas ha crecido en los últimos años debido al aumento de la población y el incremento de la congestión vehicular, que se dan en las grandes ciudades, dificultando el logro de objetivos comunes entre los diferentes actores en DUM entre otros, por la alta complejidad que se presenta. Para este artículo, se realizó una revisión amplia y actualizada en la literatura científica sobre el tema, posteriormente se realizó una categorización de los modelos utilizados en la DUM y las técnicas utilizadas en estos modelos, por último se presentan las tendencias actuales y posibles soluciones futuras en el modelado de los problemas en esta área de investigación.

PALABRAS CLAVE: Distribución urbana de mercancía; Logística de ciudad; Modelos logísticos.

\section{LOGISTICS MODELS APPLIED IN THE URBAN GOODS DISTRIBUTION}

\section{ABSTRACT}

This paper shows a classification of logistic models that have been developed to solve the problems of integration, coordination, sustainability and mobility that arise in the urban goods distribution (DUM). The search for improvement

1 Director del Grupo I+D+I Logística Industrial - Organizacional "GICO". Profesor Titular, Facultad de Minas, Universidad Nacional de Colombia.

2 Ingeniero Industrial, Magister en administración, estudiante Doctorado en Ingeniería, Industria y Organizaciones. Universidad Nacional de Colombia - Sede Medellín.

3 Ingeniero industrial. Magister en ingeniería administrativa. Doctorado en Ingeniería Industria y Organizaciones. Universidad de San Buenaventura.

Autor de correspondencia: Gómez Martínez, C.G. (Cristian Giovanny): Carrera 80 No 65-223, Universidad Nacional de Colombia, Bloque M8B. 9903. Campus Robledo, Medellín, Colombia. Tel.: 57-3006106153. Correo electrónico: crggomezma@unal.edu.co
Historia del artículo:

Artículo recibido: 05-I-2017 / Aprobado: 20-III-2018

Disponible online: 30 de marzo de 2018

Discusión abierta hasta abril de 2019 
and optimization of the processes in the distribution systems of goods and services in urban areas has spread out in recent years due to population growth and the increment of vehicular congestion that occur in large cities, making difficult the achievement of common objectives among the different actors in DUM among others, due to the high complexity that is presented. For this paper, a broad and updated review was made in the scientific literature on the subject, afterwards a categorization of the models used in the DUM and the techniques used in these models was carried out, finally presenting the current tendencies and possible future solutions in the modeling problems in this research area.

KEYWORDS: Urban goods distribution, City logistics, Logistics Models.

\section{MODELOS LOGÍSTICOS APLICADOS NA DISTRIBUIÇÃO URBANA DE MERCADORIAS}

\section{RESUMO}

Este artigo apresenta uma classificação dos modelos logísticos que foram desenvolvidos para resolver os problemas de integração, coordenação, sustentabilidade e mobilidade que surgem na distribuição urbana de mercadorias (DUM). A busca pela melhoria e otimização de processos em sistemas de distribuição de bens e serviços em áreas urbanas tem crescido nos últimos anos devido ao crescimento populacional e aumento do congestionamento do tráfego, que ocorrem nas grandes cidades, dificultando a realização de objetivos comuns entre os diferentes intervenientes nas DUM entre outros, a alta complexidade apresentada. Para este artigo, uma revisão abrangente e atualizado foi realizado na literatura científica sobre o assunto, em seguida, uma categorização dos modelos utilizados na DUM e técnicas utilizadas nestes modelos foi realizada, finalmente, as tendências atuais e possíveis soluções futuras são apresentados modelagem dos problemas nesta área de investigação.

PALAVRAS-CHAVE: distribuição urbana de mercadorias; logística da cidade; modelos logísticos.

\section{INTRODUCCIÓN}

La distribución urbana de mercancías se ha visto afectada por el aumento de la población mundial que vive en zonas urbanas, ya que esta se ha incrementando paulatinamente, como lo reflejan las cifras del Departamento de Economía y Asuntos Sociales de las Naciones Unidas donde muestran que en el año 2014 el 54\% del total de la población estaba establecida en zonas urbanas y se espera que para el año 2050 esta cifra sea del 66\% (United Nations Department of Economic and Social Affairs, 2014). El anterior crecimiento en la población urbana en el mundo genera diferentes retos para la gestión de las ciudades, como: dar respuesta al incremento del intercambio mercancías, servicios, medios de transporte para carga y semovientes, la movilidad urbana de personas e intercambio de información. Todo lo anterior afecta la calidad de vida de los ciudadanos, haciendo que sea un elemento prioritario disminuir los impactos urbanos y medio ambientales que se generan en la DUM.

Las conformaciones de distribución de carga en las ciudades se ven afectadas directamente por los sistemas logísticos, dichos impactos se pueden evidenciar en las investigaciones sobre logística de ciudad que ha crecido en los últimos años; por ejemplo (Camargo et al., 2015) realizan una revisión de los sistemas de transporte de pasajeros con enfoque 
multicriterio entre 1982 y 2014 donde se evidencia como se puede ayudar a la toma de decisiones de los sistemas de transporte teniendo en cuenta tanto los problemas económicos, como ambientales y los sociales, por su parte (Figliozzi, 2010) presenta como se ve afectado el costo, el tiempo promedio de viaje y su variabilidad en los vehículos comerciales en la ciudad por la congestión que se vive en las calles, (Woudsma et al., 2008) señalan como los sistemas logísticos pueden permitir establecer políticas de uso de la tierra en las ciudades para fines logísticos, a partir de evaluar los costos del sistema de transporte y el desarrollo del uso de la tierra, la accesibilidad, la complejidad de la red de distribución y los tiempos de flujos en la red.

(Behrends, 2016) expone los diferentes sectores económicos que se ven afectados por la logística de ciudad como lo son el detallista, los transportadores, las empresas de mensajería y paquetería, los hoteles y restaurantes, la construcción, la recolección de basuras, y transporte industrial, (Dablanc, 2007) establece que el transporte de mercancías debe unir a los transportadores y la administración pública para planear las rutas y establecer las reglas claras para el acceso de los vehículos de carga a las ciudades, (Tadić, Zečević y Krstić, 2015) afirma que la logística de ciudad impacta directa o indirectamente el crecimiento de la infraestructura de carreteras, los planes espaciales de la ciudad, las políticas o leyes de regulación de tráfico, los impactos ambientales, las condiciones de tráfico entre otros, (Taniguchi, Thompson y Yamada, 2014) presentan los últimos desarrollos y tendencias en logística de ciudad con el fin de hacer las ciudades mas sostenibles y habitables.

Una posible estrategia que plantean algunos autores para solucionar los actuaciones mencionados anteriormente es la logística de ciudad, definida según Taniguchi, et al., (2001) como "el proceso para la optimización total de las actividades logísticas y de transporte de compañías privadas con el apoyo de sistemas de información avanzado en áreas urbanas considerando el ambiente, la congestión y la seguridad en el tráfico y los ahorros de energía dentro del contexto de una economía de mercado". De acuerdo a la definición anteriormente mencionada, la logística de ciudad debe ser una iniciativa de la empresa privada y contar con sistemas de información que ayuden a la toma de decisiones de todas las partes que intervienen en ella, por lo cual no debe ser aislada, mas bien debe estar integrada a las autoridades locales con el fin de ayudar en la sostenibilidad de la ciudad.

Uno de los principales campos de análisis en la logística de ciudad es la distribución urbana de mercancías (DUM), orientada al transporte de carga en áreas urbanas para conectar la oferta y la demanda de productos en las ciudades y todos los servicios necesarios para realizar de dicha actividad, teniendo en cuenta características propias del contexto como la congestión vehicular, las políticas restrictivas de movilidad en cada municipalidad, el impacto al medio ambiente (Barceló, Grzybowska y Pardo, 2007), entre otras. En la DUM se presentan oportunidades para mejorar la calidad de vida en las ciudades, así como la integración de los diferentes actores y de los diferentes flujos que estos generan para mitigar la congestión vehicular, los accidentes y la contaminación y mejorar el desempeño del subsistema logístico de ciudad en la recolección y entrega de mercancias con el fin de dar respuestas eficientes a los clientes (Malhene et al., 2012); estas son algunas razones por las cuales se busca la optimización de procesos al interior de la DUM.

Para abordar la problemática antes mencionada, este artículo se organiza de la siguiente manera: en la sección dos se define el concepto de distribución urbana de mercancías (DUM) y los problemas más representativos en esta área de estudio. La sección tres se aborda las estrategias para enfrentar estos problemas. En la sección cuatro se realiza una clasificación de los modelos utilizados en las estrategias. En la sección cinco se presentan las tendencias en este tema y por último se finaliza con las conclusiones. 


\section{DISTRIBUCIÓN URBANA DE}

\section{MERCANCÍAS (DUM)}

La distribución urbana de mercancía hace parte de la logística de ciudad y es "el último eslabón de servicio en la cadena de transporte" también conocido como último kilometro, e incluye todos los movimientos relacionados con el comercio, el suministro y la distribución de productos en las ciudades (Antún, 2013), cuyo objetivo final es mejorar la eficiencia del sistema de transporte urbano de mercancías a la vez que reduce los impactos en el medio ambiente y la congestión vehicular (Arango-Serna et al., 2012).

Además, la DUM genera mejoras en los procesos de entrega y recogida de mercancía, en los efectos negativos en la movilidad, y en el medio ambiente dentro de las ciudades, (Crainic, Ricciardi y Storchi, 2004; Sanz, Pastor y Benedito, 2013) por lo cual es un tema de especial importancia para la planeación urbana (Tadić, Zečević y Krstić, 2015), pero de acuerdo Behrends, Lindholm y Woxenius, (2008) las autoridades administrativas de las ciudades no lo tienen en cuenta dentro de la misma, como si lo hacen con el transporte de pasajeros.

La DUM implica un sistema complejo que incluye elementos de transporte, planeación urbana y de infraestructura, las estrategias logísticas de los despachadores, los receptores o clientes y los transportadores, además de tener en cuenta la congestión, las normas de tránsito y restricciones vehiculares, entre los cuales existe un intercambio permanente de información y de productos; lo cual exige que los diferentes actores pertenecientes a la DUM deban trabajar con una metodología integrada y coordinada para lograr resultados eficientes (Bozzo, Conca y Marangon, 2014).

En la Figura 1 se puede observar que los diferentes actores principales de la DUM como son despachadores, transportadores, clientes y administradores de la ciudad, se interrelacionan entre sí, de igual manera, todos ellos aportan a los problemas de medio ambiente en la ciudad, utilizan recursos como la infraestructura y las tecnologías de la información y comunicaciones (TIC's), por lo cual una de la ideas de la DUM es buscar que trabajen de forma conjunta y tener información actualizada del tráfico de la ciudad con el fin de realizar eficaz y eficientemente la entrega o recolección de la mercancía impactando de la menor manera el medio ambiente en la ciudad.

Figura 1. Actores y contexto de la Distribución Urbana de Mercancías. Fuente: Elaboración propia

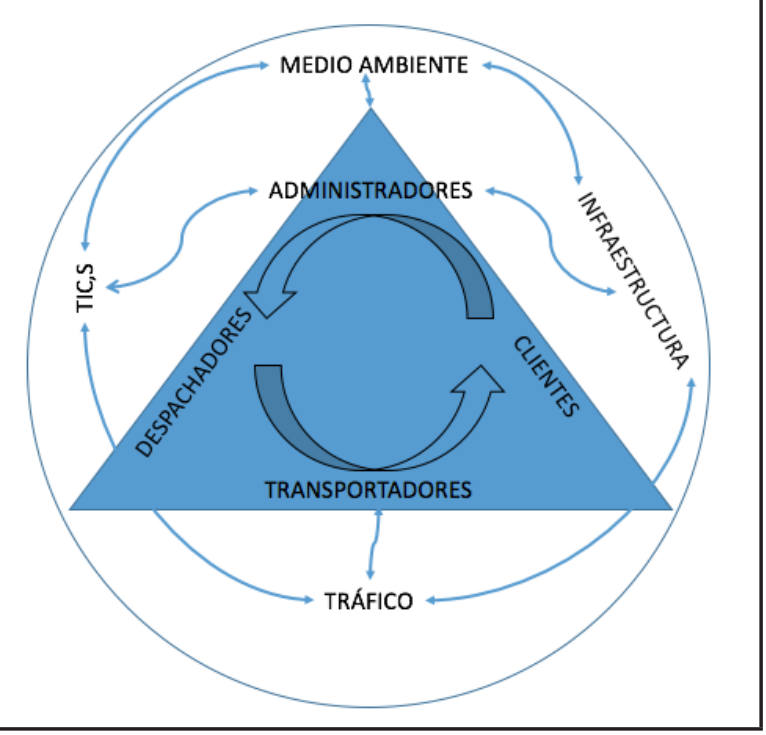

Una de las características principales de la DUM es que posee un gran componente dinámico, debido al constante movimiento de los vehículos en la ciudad y lo que esto conlleva, la variación en los tiempos de tránsito debido a posibles accidentes, a la acumulación de flujo vehicular, así como en un momento dado, la poca circulación de vehículos en las vías (Chen, Hsueh y Chang, 2006; Ehmke, Steinert y Mattfeld, 2012), pero adicionalmente, también está presente una variación en los tiempos de atención por parte de los clientes (Kuo, Wibowo y Zulvia, 2016; Yang et al., 2016), que puede ser por la cantidad de vehículos que esperan ser atendidos o simplemente por demoras en la atención a los vehículos, y también se encuentran la naturaleza estocástica de la demanda con la posible llegada o cancelación de pedidos (Li, Mirchandani y Borenstein, 2009; Mańdziuk and Żychowski, 2016); todo esto 
puede producir un cambio en los planes de distribución que se habían diseñado al inicio de la jornada.

La compleja interacción entre cada uno de los actores de la DUM y la búsqueda de un objetivo en común; y además las diferentes dinámicas que se presentan en el entorno de la logística de ciudad conllevan a que se presenten problemas en integración, coordinación, sostenibilidad y movilidad, los cuales se tratan en las siguientes secciones.

\subsection{Problemas logísticos en la distri-} bución urbana de mercancías

El manejo eficiente y sostenible de la DUM exige una coordinación e integración entre los diferentes actores que participan en ella, ya que cada uno de estos tiene diferentes objetivos que deben converger en un lugar en común para que las soluciones sean aceptables para todos. Cabe destacar los objetivos generales de la logística de ciudad, los cuales buscan alcanzar sostenibilidad, movilidad y habitabilidad por medio del balance entre el crecimiento de la economía en las ciudades y un ambiente más silencioso y tranquilo (Taniguchi, 2015).

La Figura 2 resume una clasificación de problemas en la DUM. En esta figura se puede apreciar tipos de problemas que se presentan, integración, coordinación, sostenibilidad y movilidad, los cuales se presentan a lo largo de toda las actividades que se realizan al interior de la DUM y además pueden darse por separado o estar presentes de manera conjun- ta, en todo el sistema de la DUM y los cuales se pretenden enfrentar por medio de modelos logísticos.

Con base en lo anterior, se sugiere una clasificación de los problemas logísticos de la distribución urbana de mercancías de la siguiente manera:

\subsubsection{Integración:}

Tiene que ver con la incorporación de todos los actores al sistema desde la adquisición de los datos hasta el ruteo de los vehículos teniendo en cuenta sus diferentes puntos de vista y objetivos perseguidos (Ehmke, 2012), las TIC's son necesarias para lograr esta integración.

\subsubsection{Coordinación:}

Los problemas de coordinación se presentan cuando existen varios recursos de uno o mas actores y deben trabajar de manera conjunta para alcanzar objetivos comunes de forma que la utilización de todos los recursos se maximicen de manera global y generen un desempeño mejorado. (Crainic, Ricciardi y Storchi, 2007) muestran la coordinación entre despachadores, transportadores y sus movimientos al igual que la consolidación de carga de diferentes clientes en sistemas logísticos de ciudad que tienen dos etapas, mientras que (Lin, 2011) desarrolla un modelo en el que se realiza la colaboración entre dos formas de entrega de un mismo transportador, con camiones livianos y pesados.

Figura 2. Clasificación de problemas de la DUM. Fuente: Elaboración propia

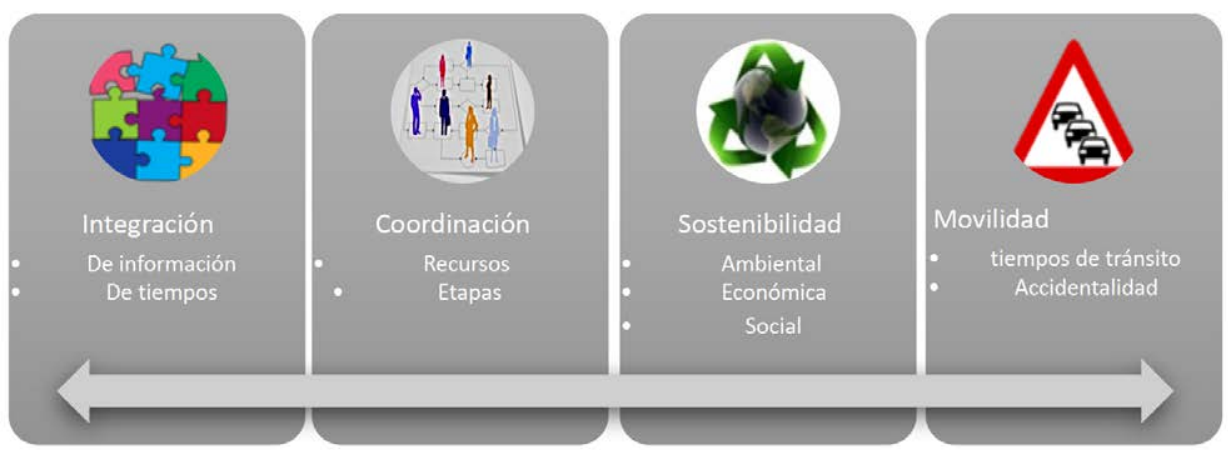




\subsubsection{Sostenibilidad:}

Se relaciona con el efecto que tiene la DUM con los tres componentes principales ambiental, social y económico y como poder disminuir los efectos secundarios de contaminación, ruido, muertes por accidentalidad, inseguridad en el tráfico local, demoras en las entregas y además aumentar la confiabilidad en la entrega (Melo, 2010).

\subsubsection{Movilidad:}

Se refieren a las dificultades de movimiento dependiendo de un sistema de transporte y de la diversidad de opciones para llegar al destino final donde las necesidades de consumo son satisfechas a un costo moderado para los transportadores y la sociedad y tan confiable en el tiempo como se pueda (Melo, 2010).

La Tabla 1 resumen de algunos de los principales trabajos orientados a resolver problemas asociados a la DUM y su característica principal de acuerdo a la clasificación anterior.

La integración se puede realizar desde el enfoque de diferentes actores de la DUM (Lindholm y Behrends, 2012; Lindawati, Gohabc y de Souza, 2014) hasta una integración que genere retos mas grandes como la de diferentes ciudades (Faccio y Gamberi, 2015). Pero se puede decir que la coordinación y la integración deben asociarse para la resolución de estos problemas, ya que no se puede generar una coordinación de recursos sin antes tener una integración de procesos e información y no se puede integrar los procesos sin pensar en la coordinación los recursos de los diferentes actores (Malhene et al., 2012). Como es el caso que expone (Castrellón-Torres et al., 2015) en el cual se utiliza como mecanismo de coordinación la consolidación de carga y administración del inventario entre productores y operadores logísticos del sector de alimentos perecederos.

Para realizar la integración y la coordinación, es necesario compartir información importante para el buen desempeño de la DUM (Muñuzuri et al., 2012) y una buena herramienta para compartir esta informa- ción son las TIC's y su uso adecuado, lo que permite la posibilidad de brindar apoyo a la creación de alianzas entre el sector público y el sector privado (Taniguchi, 2014), en este caso, la administración municipal y los despachadores y transportadores. Otro tipo de información que toma especial atención en la distribución urbana de mercancías es la referida a las variables dependientes del tiempo que mediante la provisión e integración de estos datos se mejora el sistema de planeación de rutas para los cuales se hace necesario nuevamente las TIC's, además de utilizar la integración entre modelos de optimización clásica y modelos de información (Ehmke, 2012).

Por su parte la sostenibilidad es una de las principales áreas de estudio en la logística de ciudad en sus tres dimensiones: ambiental, social y económica. Teniendo en cuenta que la eficiencia de la DUM puede estar afectada por la congestión vehicular que impacta directamente en la movilidad (Melo, 2010).

Los inconvenientes en la movilidad traen retraso en las entregas, incremento en los costos asociados a: los retardos, los tiempos de tránsito, a los pagos de horas extra a los conductores, los combustibles, a la penalización por incumplimientos y confiabilidad (Figliozzi, 2010).

Tanto la integración como la coordinación pueden integrarsen para resolver problemas de movilidad y sostenibilidad. Lo anterior se puede evidenciar con la propuesta que realiza Faccio y Gamberi (2015), donde ellos realizan la integración de un conjunto de ciudades pequeñas cercanas entre sí, con el fin de recibir la carga proveniente de diferentes partes y por medio de la coordinación de dos tipos de vehículos y una plataforma logística se lograr realizar la entrega en cada ciudad aportando a la disminución de emisiones, ya que la última parte de la distribución la realizan con vehículos eléctricos. Por su parte (Gonzalez-Feliu y Morana, 2014) muestran como la participación de diferentes tomadores de decisiones en la DUM pueden ayudar a tener una mejor medición de los impactos que se generan con el fin de mejorar la sostenibilidad del sistema. 
TABLA 1. CLASIFICACIÓN DE PROBLEMAS EN LA DUM. FUENTE: ELABORACIÓN PROPIA

\begin{tabular}{|c|c|c|}
\hline Autor (s) & Problema & Característica \\
\hline (Behrends, Lindholm y Woxenius, & Sostenibilidad & Implicar a todos los actores con la sostenibilidad \\
\hline (Melo, 2010) & $\begin{array}{l}\text { Sostenibilidad y } \\
\text { movilidad }\end{array}$ & $\begin{array}{l}\text { Impacto en la calidad del ambiente urbano y la } \\
\text { eficiencia de la DUM }\end{array}$ \\
\hline (Figliozzi, 2010) & Movilidad & $\begin{array}{c}\text { Análisis de la congestión y los costos asociados para el } \\
\text { transportador en la DUM }\end{array}$ \\
\hline (Lindholm y Behrends, 2012) & $\begin{array}{l}\text { Integración y } \\
\text { Sostenibilidad }\end{array}$ & Planeación integrada del transporte urbano \\
\hline (Malhene et al., 2012) & $\begin{array}{l}\text { Integración y } \\
\text { Coordinación }\end{array}$ & Interoperabilidad, centros de consolidación de carga \\
\hline (Ehmke, 2012) & Integración & Modelación de la integración de la información \\
\hline (Muñuzuri, et al., 2012) & Coordinación & Falta de información, políticas aisladas \\
\hline $\begin{array}{l}\text { (Palacio-Leon y Adarme-Jaimes, } \\
\text { 2014) }\end{array}$ & Coordinación & $\begin{array}{l}\text { Intervalo económico de pedido para múltiples ítems y } \\
\text { empresas }\end{array}$ \\
\hline (Gonzalez-Feliu y Morana, 2014) & Sostenibilidad & Participación del sector público y privado \\
\hline (Taniguchi, 2014) & Sostenibilidad & $\begin{array}{c}\text { Aplicación de las TIC's, cambio de mentalidad y } \\
\text { alianzas público-privadas }\end{array}$ \\
\hline $\begin{array}{l}\text { (Taniguchi, Thompson y Yamada, } \\
\text { 2014) }\end{array}$ & Sostenibilidad & $\begin{array}{l}\text { Emisiones de gases, problemas del sector salud y } \\
\text { megaciudades }\end{array}$ \\
\hline (Lindawati, Gohabc y de Souza, 2014) & Integración & $\begin{array}{l}\text { Iniciativas propias de los actores y factores que } \\
\text { influyen en la colaboración }\end{array}$ \\
\hline (Schliwa et al., 2015) & Sostenibilidad & Vehículo alternativo (moto-cargos, bicicletas) \\
\hline (Fatnassi, Chaouachi y Klibi, 2015) & Sostenibilidad & Transporte compartido de mercancías y de pasajeros \\
\hline (Faccio y Gamberi, 2015) & $\begin{array}{l}\text { Integración, } \\
\text { Coordinación y } \\
\text { Sostenibilidad }\end{array}$ & $\begin{array}{l}\text { Integración de ciudades para el manejo de la carga } \\
\text { con camiones con vehículos eléctricos }\end{array}$ \\
\hline (Castrellón-Torres et al., 2015) & Coordinación & Consolidación de carga y coordinación del inventario \\
\hline $\begin{array}{l}\text { (Taniguchi, Thompson y Yamada, } \\
\text { 2016) }\end{array}$ & Sostenibilidad & Tendencias en la logística de ciudad \\
\hline
\end{tabular}


Finalmente para afrontar y dar posible soluciones a las diferentes situaciones anteriormente enunciadas, se proponen algunas estrategias que pueden dar una solución factible a cada situación; dichas estrategias se estudiaran con mayor detalle en el siguiente numeral.

\section{ESTRATEGIAS PARA ENFRENTAR}

\section{LOS PROBLEMAS EN LA DUM}

Con el fin de entender y mejorar el sistema de la distribución urbana de mercancías se han desarrollado diferentes estrategias, pero cabe destacar que las características particulares de cada ciudad tienen una relación directa con la estrategia seleccionada para la DUM (Antún, 2013; Domínguez, 2013; Tadić, Zečević y Krstić, 2015) pues cada ciudad cuenta con características distintas que la hacen diferenciarse de las demás, por ejemplo no es lo mismo realizar el proceso de distribución en ciudades grandes que en ciudades pequeñas o en ciudades con centros históricos que sin ellos. Aún con estas diferencias las estrategias pueden ser divididas en dos grupos para buscar soluciones a los problemas, estos grupos son: 1) desde el punto de vista de la administración y políticas públicas (Sanz, Pastor y Benedito, 2013), 2) iniciativas desde el modelado del sistema (Crainic, Ricciardi y Storchi, 2007).

\section{Figura 3. Estrategias para la solución de problemas de la} DUM. Fuente: Elaboración propia

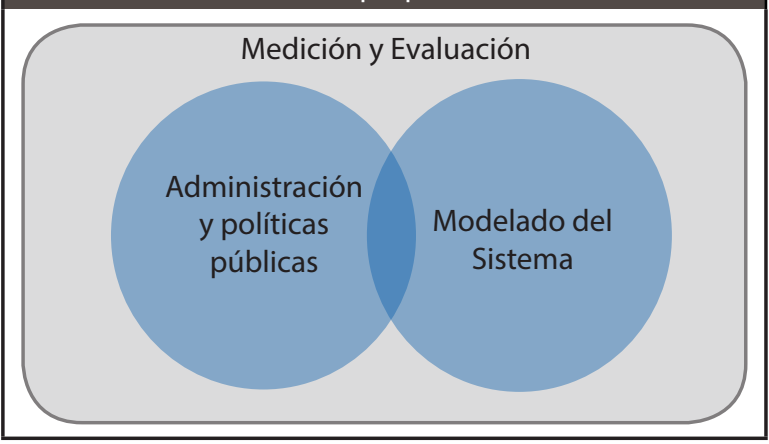

En la Figura 3 se puede observar una clasificación de los diferentes grupos de estrategias y que estas tienen un punto de intercepción donde se pue- den unir ambos, es de donde surge la iniciativa de aplicar tanto estrategias de políticas públicas como las de modelado del sistema, de este modo se pueden analizar desde diferentes frentes los problemas para dar una solución factible de manera mas integral donde los diferentes actores de la DUM puedan tener una participación más activa que facilite la evaluación permanente de dichas estrategias.

A continuación se detallan las estrategias anteriormente mencionadas, sus características y algunas aplicaciones reales.

\subsection{Estrategias de la Administración y Políticas Públicas}

Desde el punto de vista de las decisiones políticas Quak \& De Koster (2009) exponen que las más usadas son las ventanas de tiempo y las restricciones vehiculares, estas son importantes ya que separa las actividades de compra de las de entrega, reduce los impactos como la polución, aumenta el atractivo de ciertas zonas de la ciudad y aumenta la calidad de vida, pero no se han realizado estudios específicos que muestren como afectan estas el desempeño del sistema logístico urbano en términos de costos. Un estudio desarrollado por Muñuzuri (2003), recopila la mayoría de soluciones que pueden realizarse desde el enfoque de medidas políticas y para las cuales son necesarias todo el apoyo de la administración pública de la ciudad, es por esto que el enfoque a las soluciones de logística urbana deben realizarse desde el nivel estratégico con el diseño y aplicación de modelos de optimización de los recursos necesarios para cumplir con el objetivo de la misma.

En este grupo de estrategias Domínguez (2013) presenta algunas medidas para regular el tráfico, las zonas de parqueo y los accesos a los vehículos dentro de las cuales se encuentran la regulación del acceso a los vehículos de reparto, zonas de bajas emisiones, aplicación de nuevas tecnologías, reparto nocturno y otras iniciativas de tiempo compartido, áreas eficientes de carga y descarga. 


\subsection{Estrategias de Modelado del Sistema}

Desde el punto de vista del modelado del sistema son muchos los modelos desde el nivel táctico y operativo que se han desarrollado para enfrentar los retos que supone la DUM. (Crainic, Gendreau y Jean-Yves, 2009) realizan una división de estos modelos en tres tipos, modelos de demanda, de suministro y de asignación.

Los modelos de demanda según Comi et al. (2012), se dividen en cuatro basados en: 1) el vehículo, 2) los productos, 3) la entrega y 4) el modo de transporte teniendo en cuenta las condiciones de congestión y polución. Los modelos de suministro representan la infraestructura del transporte y los servicios necesarios para su operación y los modelos de asignación están enfocados en los flujos multiproductos para redes multimodales (Crainic, Gendreau y Jean-Yves, 2009).

$\mathrm{Al}$ interior de este grupo de estrategias se pueden incluir las expuestas por Domínguez (2013) como la consolidación de esquemas y medidas dirigidas a mejorar la cadena de suministro en ámbito urbano como lo son los espacios logísticos urbanos, puntos de recogida, centros de consolidación urbana.

De igual forma Domínguez (2013) también presenta otras estrategias en las cuales se encuentra los sistemas inteligentes de transporte, estrategias de planificación, procesos de consulta y esquemas de etiquetado, las cuales pueden pertenecer a las dos clasificaciones anteriores.

Los grupos de estrategias se pueden desarrollar de manera aislada o de manera conjunta, pero lo más conveniente es que se pueda realizar de manera conjunta, ya que la asociación de los actores públicoprivados ayudan a generar un impacto mayor de las estrategias en la resolución de los problemas (Russo y Comi, 2011; Gammelgaard, 2015; Taniguchi, 2015).

Para la aplicación de estas estrategias y su evaluación en el desempeño del sistema de la DUM es necesario realizar el modelado y la optimización de las mismas, por lo cual en el siguiente numeral, se desarrolla una clasificación de los modelos desarrollados para implementar dichas estrategias.

\section{MODELOS PARA LA DISTRIBUCIÓN URBANA DE MERCANCÍAS}

La DUM necesita herramientas de apoyo a la toma de decisiones que permitan mejorar su desempeño y a la vez tratar con los efectos secundarios que se producen y que formen el sistema de apoyo a las decisiones, los cuales están basados principalmente en el modelado, la optimización, la simulación y procedimientos de evaluación, (Deflorio et al., 2012).

La DUM implica el modelamiento y la optimización como parte de un conjunto de retos y oportunidades para realizar desarrollos metodológicos con un impacto social real (Crainic, Ricciardi y Storchi, 2009), dado que se abordan diferentes herramientas existentes en la investigación de operaciones y los sistemas de transporte dentro el contexto urbano para dar respuesta a los problemas que se presentan y mejorar de manera eficiente la distribución urbana de mercancías.

Es por ello que el modelado y la optimización se convierten en herramientas para dar soluciones a los problemas que presenta el transporte urbano de carga (Ehmke, 2012), pero el modelador se enfrenta a problemas específicos por el contexto del sistema que se va modelar, algunos de los cuales se presentan a continuación:

\subsection{Problemas de Modelamiento de Ia DUM}

El modelado de la administración de los vehículos en la DUM corresponde a problemas de optimización combinada que son difíciles de resolver o que tomarían demasiado tiempo resolverlos de forma exacta, además se deben tener presente los requerimientos de tiempo real necesarios para solucionar los problemas (Crainic, Gendreau y Jean-Yves, 2009). Una de las dificultades que se encuentra al realizar el modelado en la DUM tiene que ver con 
la gestión de la incertidumbre en la demanda, debido a que se puede realizar la planeación de la distribución a los clientes pero pueden llegar nuevos clientes mientras se realiza el recorrido; también se puede presentar incertidumbre en los tiempos de viaje, y esta es causada por las diferentes situaciones de tráfico que se pueden presentar (Yan, Lin y Lai, 2013; Zufferey, Cho y Glardon, 2016). Igualmente en el modelado la calidad de la información es fundamental, toda vez que se debe tener en cuenta el momento exacto en el que se ajusta o calcula una variable (Chac Sam, 2010).

Teniendo en cuenta los anteriores inconvenientes que se pueden presentar en el modelado, en este documento se propone una clasificación de los modelos para la DUM: modelos de demanda, de ruteo para la planeación, selección y evaluación de políticas.

\subsubsection{Modelos de demanda:}

Los modelos de demanda buscan identificar los productores, transportadores, despachadores, clientes, el nivel de consumo, los productos que se mueven, los volúmenes de distribución desde origen hasta destino (Crainic, Gendreau y Jean-Yves, 2009). Estos modelos son importantes ya que se pueden conocer cuál es el comportamiento de la demanda de acuerdo a varios factores o mediciones entre las cuales están la cantidad de carga movida entre un origen y un destino, los viajes de entrega que se realizan y en que tipo de vehículo para poder escoger las alternativas de entregas de mercancías (Nuzzolo y Comi, 2014). Estimar la cantidad de producto que se mueven puede ser complicado debido a las paradas múltiples en partes diferentes de la ciudad, cambio de rutas entre días, por lo cual la estimación de la matriz origen destino también puede ser complicada (Muñuzuri et al., 2009).

De acuerdo con Nuzzolo y Comi (2014), el modelo de demanda posee tres submodelos de la siguiente manera:
Submodelo de cantidad que permite estimar la cantidad de matrices origen destino de acuerdo con el tipo de carga y el tipo de transporte

$$
Q_{o d}[r]=Q_{d} \cdot p[o / d] \cdot p[r / o d]
$$

Submodelo de envíos que son los promedios de flujo entregado en la matriz O-D llevado por un transporte tipo $r$ sobre una pareja O-D en un periodo de tiempo de entrega $\tau$

$$
N D_{o d}[\tau r]=Q_{o d}[r] \cdot p[\tau / d] / q[r]
$$

Submodelo del vehículo que obtiene las matrices O-D usando un modelo de recorrido de entrega multi-etapa analítico agregado que considera el promedio de comportamiento de los reabastecedores.

$$
\mathrm{T}_{0}[\mathrm{vnt \tau r}]=\mathrm{T}_{0}[\tau \mathrm{\tau r}] \cdot \mathrm{p}[\mathrm{t} / \mathrm{\tau ro}] \cdot \mathrm{p}[\mathrm{n} / \mathrm{t \tau ro}] \cdot \mathrm{p}[\mathrm{v} / \mathrm{nt \tau ro}]
$$

Donde

$Q_{o d}[r]$ es el promedio de cantidad de flujo de carga atraído por una zona $d$ llegando desde una zona 0 con un tipo de transporte $r$;

$Q_{d}$ es el promedio de cantidad de carga atraída por una zona $d$ obtenido por el modelo de atracción;

$p[o / d]$ la probabilidad que una carga sea atraída a una zona d llegando desde una zona 0 ;

$p[r / o d]$ es la probabilidad de ser reabastecido por un tipo de transporte $r$ obtenido por un modelo de tipo de transporte de elección discreta;

$N D_{o d}[\tau r]$ es el número de entregas realizadas por un transporte tipo $r$ sobre un par origen-destino en un tiempo de entrega $\tau$;

$p[\tau / d]$ es la probabilidad de tener entregas en el destino del minorista en un tiempo de entrega $\tau$ obtenido por un modelo de periodo de entrega;

$q[r]$ es el promedio de cantidad de carga entregada con un transporte tipo $r$;

$\mathrm{T}_{0}$ [vnttr] El número de viajes con $n$ paradas para entregas que parten de la zona de origen 0 en 
el momento t del período de entrega $\tau$ y operados por el transporte $r$ y el tipo de vehículo $v$;

$\mathrm{T}_{0}[\tau r]$ es el número total de viajes que salen de la zona de origen 0 en el periodo de tiempo $\tau$;

$p[t / \tau r o]$ es la probabilidad que el viaje de entrega salga en un tiempo dado $\tau$ de un origen 0 obtenido por un modelo de tipo de transporte de elección discreta;

$\mathrm{p}[\mathrm{n} / \mathrm{t}$ tro] es la probabilidad que las entregas sean realizadas por un viaje saliendo desde una zona 0 con $n$ paradas obtenidas por un modelo de elección de cadena de viajes discreto;

$\mathrm{p}[\mathrm{v} / \mathrm{nt \tau ro}]$ es la probabilidad que las entregas sean realizadas por un vehículo tipo $v$ obtenida por un modelo de elección de tipo de vehículo discreto

\subsubsection{Modelos de ruteo:}

Estos modelos incluyen la regulación del tráfico, la congestión vehicular, las condiciones de las vías, los espacios de estacionamiento, la contaminación del aire, de ruido, emergencias, las ganancias económicas y busca incluir a todos los actores del sistema aunque no siempre se logre (Kim et al., 2015) y se puede dar en una o varias etapas las cuales se tratan diferente en el modelado (Crainic, Ricciardi y Storchi, 2009), para profundizar en este tipo de modelos se puede leer (Taniguchi et al., 2001). Estos tipos de modelos tiene muchas variaciones entre las que se pueden encontrar modelado de problemas de ruteo de vehículos con capacidad limitada, con ventanas de tiempo, modelado de localización y ruteo, de inventario en el ruteo, ruteo dinámico, entre otros.

El modelo básico de ruteo VRP se puede apreciar en (Kim et al., 2015) y está representado de la siguiente manera:

$\operatorname{Min} \sum_{i \in V} \sum_{j \in V} c_{i j} X_{i j}$

Sujeta a:

$$
\begin{aligned}
& \sum_{i \in V} x_{i j}=1, \forall j \in \bigvee\{0\} \\
& \sum_{i \in V} x_{i j}=1, \forall i \in \bigcap\{0\}
\end{aligned}
$$$$
\sum_{i \in V} x_{i 0}=K
$$$$
\sum_{j \in V} x_{0 j}=K
$$$$
\sum_{i \notin S} \sum_{j \in S} x_{i j} \geq r(s), \forall S \subseteq V\{0\}, S \neq 0
$$$$
x_{i j} \in\{0,1\}, \forall I, J \in V
$$

(5) Solo un arco sale de cada nodo

(6) Solo un arco llega a cada nodo

(7) los vehículos salen inicialmente del depósito

(8) Todos los vehículos deben volver al depósito

(9) Restricciones de capacidad del vehículo (10) Restricción de variable binaria

\subsubsection{Modelos para la planeación, selección y evaluación de políticas:}

Este tipo de modelos sirven para tomar decisiones acerca de las posibles opciones que se pueden seleccionar para las políticas adecuadas en la logística de ciudad (Taniguchi, Thompson y Yamada, 2016), y además debe tener en cuenta los actores que participan en la DUM para poder clasificar el impacto que cada uno de ellos genera al desarrollar diferentes políticas (Bozzo, Conca y Marangon, 2014), una de las herramienta que se utilizan son los modelos de toma de decisiones multicriterio (MCDM por sus siglas en ingles) igual manera se pueden utilizar para evaluar los resultados de dichas políticas. Entre estos modelos se destaca el propuesto por (Bozzo, Conca y Marangon, 2014) como un modelo de evaluación anticipada de políticas que se puede apreciar en la Figura 4.

La Tabla 2 resume una clasificación de algunos modelos logísticos en la distribución urbana de mercancías, los objetivos principales que persigue y las técnicas de solución empleadas. 
Figura 4. Modelo de evaluación previa para la planeación y selección de políticas. Fuente (Bozzo, Conca y Marangon, 2014)
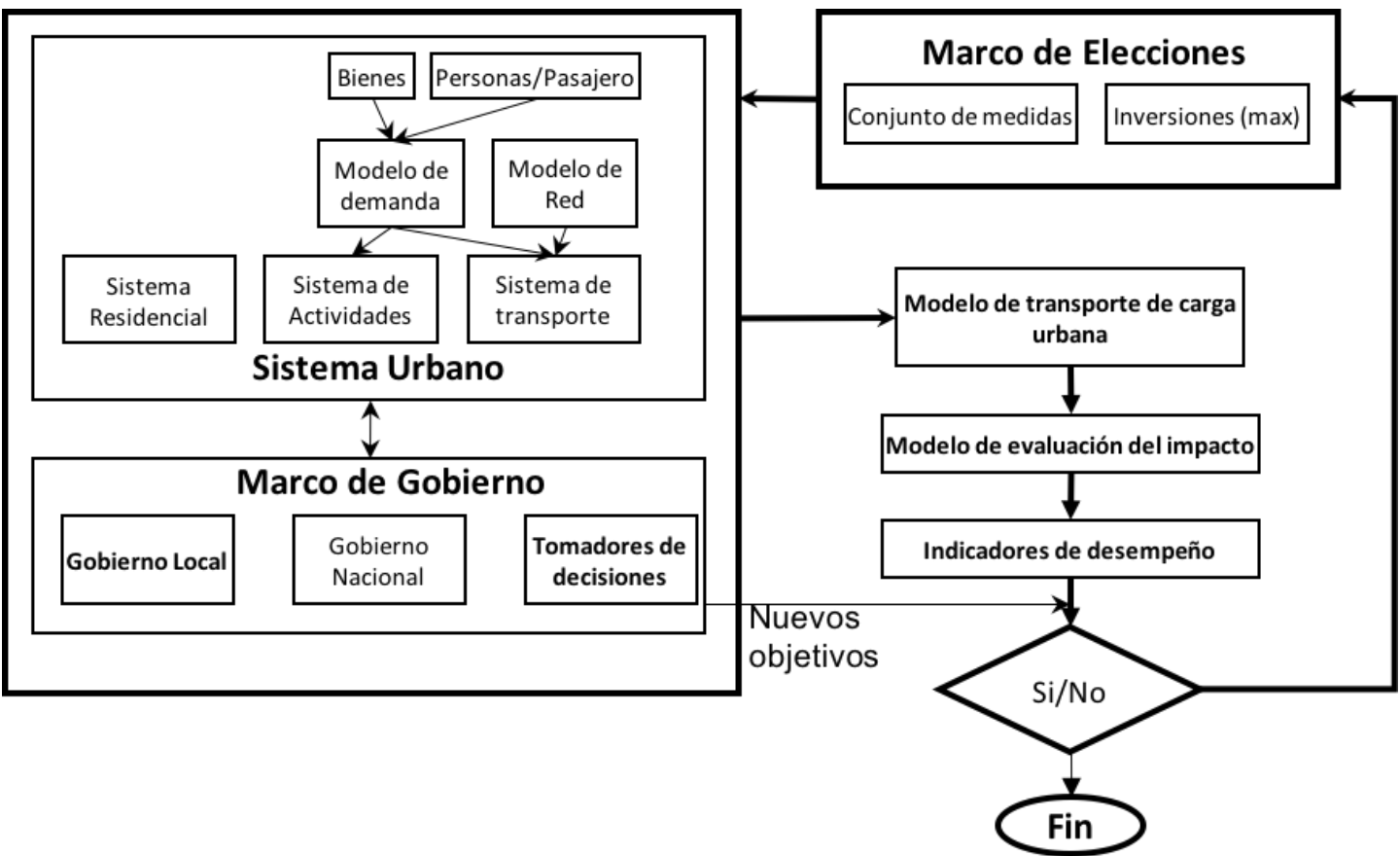

TABLA 2. TIPOS DE MODELOS LOGÍSTICOS EN LA DUM. FUENTE: ELABORACIÓN PROPIA

\begin{tabular}{|c|c|c|}
\hline Autor (s) & Clasificación & Característica del modelo y método de solución \\
\hline (Muñuzuri et al., 2009) & Modelos de demanda & $\begin{array}{c}\text { Matriz origen-destino basado en la maximización } \\
\text { de la entropía }\end{array}$ \\
\hline $\begin{array}{l}\text { (Qureshi, Taniguchi y Yamada, } \\
\text { 2009) }\end{array}$ & Modelos de ruteo & $\begin{array}{c}\text { Programación y ruteo de vehículos con } \\
\text { ventanas de tiempo. Generación de columna } \\
\text { y algoritmo branch and price }\end{array}$ \\
\hline $\begin{array}{l}\text { (Ambrosini, Patier y Routhier, } \\
\text { 2010) }\end{array}$ & Modelos de demanda & $\begin{array}{l}\text { Estudio de la demanda, encuestas y Simulación del } \\
\text { sistema de transporte de carga urbana }\end{array}$ \\
\hline (Boussier et al., 2011) & $\begin{array}{l}\text { Modelos para la } \\
\text { planeación, selección y } \\
\text { evaluación de políticas }\end{array}$ & $\begin{array}{c}\text { Simulación multiagente para optimizar los la política de } \\
\text { parqueaderos a nivel temporal y espacial }\end{array}$ \\
\hline (Brito, 2011) & Modelos de ruteo & $\begin{array}{c}\text { Optimización de ruteo de vehículos con restricciones } \\
\text { difusas. Hiperheurísticas }\end{array}$ \\
\hline (Russo y Comi, 2011) & $\begin{array}{l}\text { Modelos para la } \\
\text { planeación, selección y } \\
\text { evaluación de políticas }\end{array}$ & $\begin{array}{c}\text { Simulación para evaluar los efectos de medidas tomadas } \\
\text { a priori en la DUM. } \\
\text { Enfoque desagregado en dos niveles (productos y } \\
\text { vehículos) }\end{array}$ \\
\hline (Deflorio et al., 2012) & $\begin{array}{l}\text { Modelos para la } \\
\text { planeación, selección y } \\
\text { evaluación de políticas }\end{array}$ & $\begin{array}{l}\text { Simulación por escenarios de la demanda sobre la red y la } \\
\text { compatibilidad de tiempo y su costo asociado. }\end{array}$ \\
\hline
\end{tabular}




\begin{tabular}{|c|c|c|}
\hline $\begin{array}{l}\text { (Hemmelmayr, Cordeau } \\
\text { y Crainic, 2012) }\end{array}$ & Modelos de ruteo & $\begin{array}{l}\text { Localización y ruteo de vehículos en un } \\
\text { sistema de dos niveles. Heurística búsqueda } \\
\text { de vecindario amplio }\end{array}$ \\
\hline (Muñuzuri, et al., 2012) & Modelos de demanda & Matriz origen-destino con simulación macroscópica \\
\hline (Nguyen, Prins y Prodhon, 2012) & Modelos de ruteo & $\begin{array}{l}\text { Localización y ruteo de vehículos en un sistema } \\
\text { de dos niveles. GRASP }\end{array}$ \\
\hline $\begin{array}{l}\text { (Qureshi, Taniguchi } \\
\text { y Yamada, 2012) }\end{array}$ & Modelos de ruteo & $\begin{array}{l}\text { Ruteo dinámico con ventanas de tiempo } \\
\text { y microsimulación }\end{array}$ \\
\hline (Davis y Figliozzi, 2013) & Modelos de ruteo & $\begin{array}{c}\text { Evaluación de utilización de camiones eléctricos } \\
\text { en la distribución teniendo en cuenta costos de } \\
\text { operación, consumo de energía, tamaño de la flota } \\
\text { y energía necesaria. }\end{array}$ \\
\hline (Faure et al., 2013) & $\begin{array}{l}\text { Modelos para la } \\
\text { planeación, selección y } \\
\text { evaluación de políticas }\end{array}$ & $\begin{array}{l}\text { Centros urbanos de consolidación y colaboración } \\
\text { entre transportadores con diseño de red, } \\
\text { combinación de escenarios, ruteo. GRASP }\end{array}$ \\
\hline (Muñoz-Villamizar et al., 2013) & Modelos de ruteo & $\begin{array}{l}\text { Localización y ruteo mediante simulación Montecarlo } \\
\text { como entrada a varias heurísticas }\end{array}$ \\
\hline (Yan, Lin y Lai, 2013) & Modelos de ruteo & $\begin{array}{c}\text { Ruteo dinámico con demanda y tiempos de viaje } \\
\text { inciertos. Heurística de variable fija y una red } \\
\text { de tiempo-espacio }\end{array}$ \\
\hline (Sun, Duan y Chen, 2014) & Modelos de demanda & $\begin{array}{l}\text { Pronósticos del crecimiento la demanda } \\
\text { del transporte de carga }\end{array}$ \\
\hline (Nuzzolo y Comi, 2014) & Modelos de demanda & $\begin{array}{l}\text { Pronóstico de demanda y Estimación de la matriz } \\
\text { O-D. Modelo multietapa }\end{array}$ \\
\hline (Tadić, Zečević y Krstić, 2014) & $\begin{array}{l}\text { Modelos para la } \\
\text { planeación, selección y } \\
\text { evaluación de políticas }\end{array}$ & $\begin{array}{l}\text { MCDM para evaluar escenarios de logística de ciudad } \\
\text { en Belgrado mediante Fuzzy AHP y Fuzzy TOPSIS }\end{array}$ \\
\hline (Arango-Serna et al., 2015) & Modelos de ruteo & $\begin{array}{c}\text { Ruteo del inventario con múltiples depósitos. } \\
\text { Algoritmo genético }\end{array}$ \\
\hline (Makhloufi et al., 2015) & Modelos de ruteo & $\begin{array}{l}\text { Ruteo dinámico con Simulación por eventos } \\
\text { discretos en tiempo real }\end{array}$ \\
\hline (Muñoz-Villamizar et al., 2015) & Modelos de ruteo & $\begin{array}{l}\text { Ruteo con vehículos de capacidad limitada } \\
\text { multidepósito con escenarios de Colaboración vs no } \\
\text { colaboración. Heurística jerárquica de dos fases }\end{array}$ \\
\hline $\begin{array}{l}\text { (Arango-Serna, Romano y } \\
\text { Zapata-Cortés, 2016) }\end{array}$ & Modelos de ruteo & $\begin{array}{c}\text { Vendor management Inventory (VMI) y ruteo } \\
\text { de vehículos. Algoritmos genéticos }\end{array}$ \\
\hline $\begin{array}{l}\text { (Awasthi, Adetiloye } \\
\text { y Crainic, 2016) }\end{array}$ & $\begin{array}{l}\text { Modelos para la } \\
\text { planeación, selección y } \\
\text { evaluación de políticas }\end{array}$ & $\begin{array}{l}\text { Selección de socios para la colaboración mediante } \\
\text { el métodos BOCR y fuzzy GRA }\end{array}$ \\
\hline $\begin{array}{l}\text { (Guedria, Malhene } \\
\text { y Deschamps, 2016) }\end{array}$ & Modelos de ruteo & $\begin{array}{l}\text { Mezcla del ruteo de vehículos con restricción de } \\
\text { capacidad y optimización del cargue de los vehículos. } \\
\text { (Área de logística Colaborativa para optimizar } \\
\text { las entregas urbanas) }\end{array}$ \\
\hline $\begin{array}{l}\text { (Holguín-Veras } \\
\text { y Sánchez-Díaz, 2016). }\end{array}$ & Modelos de demanda & $\begin{array}{l}\text { Demanda administrada por el receptor. } \\
\text { Microsimulación }\end{array}$ \\
\hline
\end{tabular}




\begin{tabular}{|c|c|c|}
\hline (Koç et al., 2016) & Modelos de ruteo & $\begin{array}{c}\text { Localización y el ruteo para medir Disminución } \\
\text { de las emisiones de } \text { CO }_{2} \text {. Metaheurística ANLS }\end{array}$ \\
\hline (Montoya-Torres et al., 2016) & Modelos de ruteo & $\begin{array}{c}\text { Comparación de escenarios para verificar } \\
\text { el desempeño de redes colaborativas o no } \\
\text { colaborativas. Heurística jerárquica para VRP } \\
\text { MultiDepósito (asignación-ruteo) }\end{array}$ \\
\hline (Zufferey, Cho y Glardon, 2016) & Modelos de ruteo & $\begin{array}{c}\text { Ruteo dinámico con demanda y tiempos de viaje } \\
\text { inciertos para recogida y entrega de mercancías. } \\
\text { Metaheurística híbrida con inserción greedy } \\
+ \text { búsqueda local + intercambios }\end{array}$ \\
\hline
\end{tabular}

Los modelos de ruteo presentan una gran variedad de enfoques especialmente en las restricciones que deben cumplirse en el mismo, desde restricciones en la capacidad de los vehículos, pasando por franjas horarias de entrega y recogida limitadas hasta restricciones difusas; al igual que la combinación con otros enfoques como el ruteo con localización y ruteo con inventario. Esta gran variedad de modelos permite un amplio margen de investigación, pero la idea general es lograr modelar lo mas cercano posible a la realidad de la DUM, es por eso que el ruteo dinámico ha tomado fuerza en la investigación.

Los modelos de demanda se basan en la realización de estudios de campo para poder encontrar el movimiento de carga entre puntos de origen y destino además de utilizar técnicas como la simulación para pronosticar el comportamiento de la demanda de carga, pero una de sus principales dificultades es la naturaleza diversa de la DUM, y sus diferentes paradas dentro de una misma ruta y las diferentes cantidades de envíos según el sector, además de la escasez de datos sobre el flujo de mercancía en las ciudades.

Otros enfoques en los modelos de demanda, lo presentan (Nuzzolo y Comi, 2014; Sun, Duan y Chen, 2014) quienes se enfocan en el pronóstico de la demanda de carga urbana, el primero realiza una mezcla de modelos basados en la cantidad, entrega y vehículo para calcular las futuras matrices Origen-Destino y el segundo a través de la utilización de datos históricos existentes y su correlación con los datos de crecimiento económico como el pro- ducto interno bruto buscan predecir la demanda futura de carga.

Los modelos para la planeación, selección y evaluación utilizan es su mayoría la evaluación por escenarios para encontrar los posibles resultados que darán las políticas y los modelos en la DUM.

De acuerdo a la clasificación que se presentó en la Tabla 2 se puede observar que los modelos más estudiados son los de ruteo y dentro de estos, gran variedad de submodelos se pueden aplicar a la distribución urbana de mercancías, es por ello que se expone las tendencias en el modelado para esta área específica en la siguiente numeral.

\section{TENDENCIAS EN EL MODELADO DE LA DISTRIBUCIÓN URBANA DE MERCANCÍAS}

En el contexto de la distribución urbana de mercancías, los modelos de demanda han sido los que han tenido mayor investigación con el fin de encontrar la matriz origen-destino con sus costos y cantidad demandada, pero en este tipo de los estudios los modelos son bien conocidos (Crainic, Ricciardi y Storchi, 2009), por lo que se encontró que una tendencia en este tipo de modelos es buscar soluciones diferentes como la consolidación de carga liderada por el cliente (Holguín-Veras and Sánchez-Díaz, 2016) y el auge de modelos que estudien las respuestas de la DUM a la demanda estocástica (Zhu et al., 2014; Zhang, Lam y Chen, 2016). 
En los modelos de ruteo, se está haciendo especial énfasis en los constantes cambios que surgen en la información tanto de demanda, como de localización, de movilidad, de tiempos de atención a los vehículos que hacen que las rutas inicialmente programadas puedan variar de acuerdo a la nueva información disponible, es por eso que el ruteo dinámico (Pillac et al., 2013) y el ruteo dependiente del tiempo hacen parte de una de las variaciones del ruteo que tienen mayor potencial de uso en la DUM, y para resolver este tipo de modelos se hace necesario la utilización de las nuevas TIC's y los sistemas inteligentes de transporte como ayuda a la recolección y comunicación de la información dinámica que se genera en este tipo de problemas, para mejorar la planeación de rutas en tiempo real.

La nueva forma de negocios que se ha desarrollado por internet en especial el B2C ha obligado a la DUM a ser cada vez más eficiente, rápida y eficaz (Taniguchi, Thompson y Yamada, 2016) para llegar al cliente en el momento que lo desea, esta ha sido otra razón que ha impulsado el ruteo dinámico y la utilización de GPS y GIS como herramientas que apoyan las decisiones de ruteo de las empresas y además para realizar planeación colaborativa de distribución (Guerlain, Cortina y Renault, 2016; Li et al., 2016).

Otra tendencia adicional es el uso de vehículos menos contaminantes para reducir tanto la emisión de gases como el ruido y a la vez la congestión vehicular, ya que también son de menor tamaño, tal es el caso del uso de vehículos eléctricos (Davis y Figliozzi, 2013; Faccio y Gamberi, 2015; Fatnassi et al., 2015; Gonzalez-Feliu y Muñuzuri, 2013; HolguínVeras y Sánchez-Díaz, 2016) o el uso de vehículos alternativos como las bicicletas de carga o los ciclo motores (Schliwa et al., 2015).

Una tendencia que surge de la unión de los sistema de transporte urbano es el uso compartido de transporte de pasajeros y de mercancías es un tema que combina los conceptos de logística de ciudad y de transporte urbano de pasajeros y que puede ser un campo de investigación novedoso para futuras investigaciones (Fatnassi, Chaouachi y Klibi, 2015; Taniguchi, Thompson y Yamada, 2016).

\section{CONCLUSIONES}

En este documento se presentan diferentes aspectos de la distribución urbana de mercancías, sus problemas, estrategias para solucionarlos y los métodos de solución clasificados de acuerdo a características en común, tanto de los problemas como de los modelos. Se muestra que los diferentes modelos buscan no solo planear mejorar el desempeño de sistema sino también para disminuir los impactos negativos que produce la distribución urbana de mercancías.

Los problemas encontrados en la literatura sobre distribución urbana de mercancías se pueden clasificar en problemas de integración, coordinación, movilidad y sostenibilidad, los primeros dos impactan directamente a los actores principales y se busca su solución para mejorar el desempeño de estos, mientras que los otros dos tienen su impacto en la comunidad en general y se tratan de disminuir al máximo el daño o los efectos colaterales que se causa en la ciudad.

Los modelos realizados para representar la distribución urbana de mercancías se pueden clasificar en modelos de demanda, con los cuales se busca encontrar las matrices de origen y destino de las cargas y a partir de estos poder realizar los modelos de ruteo que generen una optimización del desempeño de los procesos de entrega y recogida de mercancías y por último se tienen los modelos para la planeación y evaluación de las políticas que se generan en las ciudades y que afectan a todos los actores del sistema.

De acuerdo a las tendencias en el modelado de la DUM, los modelos de ruteo dinámico han tenido gran acogida en la comunidad académica para su aplicación a casos cada vez más cercanos a la realidad. El modelamiento dinámico de ruteo de vehículos es un campo de atención que ayuda a cumplir con las diferentes situaciones constantemente cambiantes que se presentan en la recolección y entrega 
de mercancías en una ciudad, este dinamismo está esencialmente enfocado en la incertidumbre de la demanda, como la llegada de ordenes no esperadas y que se deben atender, y en la variación en los tiempos de tránsito, pero no se han encontrado publicaciones que estudien el efecto combinado del dinamismo por demanda, por tiempos de tránsito y por tiempos de servicio en la planeación inicial de las rutas de distribución con el fin de cumplir con la satisfacción de los clientes y a la vez tener un desempeño óptimo del sistema de distribución urbano de mercancías.

\section{AGRADECIMIENTOS}

Los autores agradecen al proyecto de investigación doctoral titulado "Modelización dinámica de distribución urbana de mercancías utilizando microsimulación multivariable e inferencia difusa." que ha dado origen a este artículo, desarrollado en el grupo de investigación I+D+i Logística Industrial - Organizacional “GICO”, en el programa Doctoral de Ingeniería - Industria y Organizaciones - adscrito a la Facultad de Minas de la Universidad Nacional de Colombia.

\section{REFERENCIAS}

Ambrosini, C., Patier, D. y Routhier, J. L. (2010) 'Urban freight establishment and tour based surveys for policy oriented modelling', Procedia - Social and Behavioral Sciences, 2(3), pp. 6013-6026. doi: 10.1016/j.sbspro.2010.04.015.

Antún, J. P. (2013) Distribución Urbana de Mercancías: Estrategias con Centros Logísticos, IDB Technical Note.

Arango-Serna, M. D., Romano, C. A. y Zapata Cortés, J. A. (2016) 'Collaborative goods distribution using the IRP model', DYNA, 83(196), pp. 204-212. doi: 10.15446/dyna.v83n196.52492.

Arango-Serna, M. D., Serna-Uran, C. A. y Alvarez Uribe, K. C. (2012) 'Collaborative autonomous systems in models of urban logistics', DYNA, 79(172), pp. 171-179.

Arango-Serna, M. D., Zapata Cortés, J. A. y Gutierrez, D. (2015) 'Modeling The Inventory Routing Problem ( IRP ) With Multiple Depots With Genetic Algorithms', IEEE Latin America Transactions, 13(12), pp. 3959-3965.
Awasthi, A., Adetiloye, T. y Crainic, T. G. (2016) 'Collaboration partner selection for city logistics planning under municipal freight regulations', Applied Mathematical Modelling, 40, pp. 510-525. doi: 10.1016/j. apm.2015.04.058.

Barceló, J., Grzybowska, H. y Pardo, S. (2007) 'Vehicle routing and scheduling models, simulation and city logistics', in Zeimpekis, V., Tarantilis, C. ., Giaglis, G. ., and Minis, I. (eds) Dynamic Fleet Management; Concepts, Systems, Algorithms and Case Studies. US: Springer, pp. 163-195. doi: 10.1007/978-0-38771722-7_8.

Behrends, S. (2016) 'Recent Developments in Urban Logistics Research - A Review of the Proceedings of the International Conference on City Logistics 2009 - 2013', Transportation Research Procedia. Elsevier B.V., 12, pp. 278-287. doi: 10.1016/j.trpro.2016.02.065.

Behrends, S., Lindholm, M. y Woxenius, J. (2008) 'The impact of urban freight transport: A definition of sustainability from an actor's perspective', Transport Planning and Technology, 31(6), pp. 693-713.

Boussier, J., Cucu, T., Ion, L. y Breuil, D. (2011) 'Simulation of goods delivery process', International Journal of Physical Distribution \& Logistics Management, 41(9), pp. 913-930.

Bozzo, R., Conca, A. y Marangon, F. (2014) 'Decision support system for city logistics: Literature review, and guidelines for an ex-ante model', Transportation Research Procedia. Elsevier B.V., 3(July), pp. 518-527. doi: 10.1016/j.trpro.2014.10.033.

Brito, J. A. (2011) Optimización de rutas de distribución con información y restricciones difusas. Universidad de Laguna.

Camargo, J., Carrillo, M. H. y Montoya-Torres, J. R. (2015) 'Multi-criteria approaches for urban passenger transport systems: a literature review', Annals of operations research, 226(1), pp. 69-87.

Castrellón, J. P., García, J. L. y Adarme Jaimes, W. (2015) 'Consolidación de carga como mecanismo de coordinación en cadenas de suministro de perecederos: Estudio de simulación Resumen', DYNA, 82(189), pp. 233-242. doi: 10.15446/dyna.v82n189.48551.

Chac Sam, A.-V. (2010) Gestión dinámica de rutas en la distribución urbana de mercancías. Universidad Politecnica de Cataluña. 
Chen, H. K., Hsueh, C. F. y Chang, M. S. (2006) 'The real-time time-dependent vehicle routing problem', Transportation Research Part E: Logistics and Transportation Review, 42(5), pp. 383-408. doi: 10.1016/j. tre.2005.01.003.

Comi, A., Site, P. D. y Filippi, F. (2012) 'Urban Freight Transport Demand Modelling : a State of the Art', European Transport/Trasporti Europei, 7(51), pp. 1-17.

Crainic, T. G., Gendreau, M. y Jean-Yves, P. (2009) 'Intelligent freight-transportation systems: Assessment and the contribution of operations research', Transportation Research Part C: Emerging Technologies. 17(6), pp. 541-557.

Crainic, T. G., Ricciardi, N. y Storchi, G. (2004) 'Advanced freight transportation systems for congested urban areas', Transportation Research Part C: Emerging Technologies, 12(2), pp. 119-137. doi: 10.1016/j. trc.2004.07.002.

Crainic, T. G., Ricciardi, N. y Storchi, G. (2007) 'Models for Evaluating and Planning City Logistics Systems', Transportation Science, 43(4), pp. 432-454. doi: $10.1287 /$ trsc.1090.0279.

Dablanc, L. (2007) 'Goods transport in large European cities: Difficult to organize, difficult to modernize', Transportation Research Part A: Policy and Practice, 41(3), pp. 280-285. doi: 10.1016/j.tra.2006.05.005.

Davis, B. A. y Figliozzi, M. A. (2013) 'A methodology to evaluate the competitiveness of electric delivery trucks', Transportation Research Part E: Logistics and Transportation Review. Elsevier Ltd, 49(1), pp. 8-23. doi: 10.1016/j.tre.2012.07.003.

Deflorio, F., Gonzalez-Feliu, J., Perboli, G. y Tadei, R. (2012) 'The influence of time windows on the costs of urban freight distribution services in city logistics applications', European Journal of Transport and Infrastructure Research, 12(3), pp. 256-274.

Domínguez, A. (2013) Modelización del comportamiento de los comerciantes ante nuevas políticas de reparto urbano de mercancías. Universidad de Cantabria.

Ehmke, J. F. (2012) Integration of Information and Optimization Models for Routing in City Logistics. Internatio. Edited by F. S. Hillier and C. C. Price. New York: Springer International Publishing. doi: 10.1007/978-1-4614-0806-2.

Ehmke, J. F., Steinert, A. y Mattfeld, D. C. (2012) 'Advanced routing for city logistics service providers based on time-dependent travel times', Journal of Computa- tional Science. Elsevier B.V., 3(4), pp. 193-205. doi: 10.1016/j.jocs.2012.01.006.

Faccio, M. y Gamberi, M. (2015) 'New City Logistics Paradigm: From the "Last Mile" to the "Last 50 Miles" Sustainable Distribution', Sustainability. Multidisciplinary Digital Publishing Institute, 7(11), pp. 14873-14894. doi: 10.3390/su71114873.

Fatnassi, E., Chaouachi, J. y Klibi, W. (2015) 'Planning and operating a shared goods and passengers on-demand rapid transit system for sustainable city-logistics', Transportation Research Part B: Methodological, 81, pp. 440-460. doi: 10.1016/j. trb.2015.07.016.

Faure, L., Battaia, G., Marques, G., Guillaume, R., Vega-Mejía, C. A., Montoya-Torres, J. R., Muñoz-Villamizar, A. F. y Quintero-Araújo, C. L. (2013) 'How to anticipate the level of activity of a sustainable collaborative network: The case of urban freight delivery through logistics platforms', in Digital Ecosystems and Technologies (DEST), 2013 7th IEEE International Conference on, pp. 126-131. doi: 10.1109/ DEST.2013.6611341.

Figliozzi, M. A. (2010) 'An iterative route construction and improvement algorithm for the vehicle routing problem with soft time windows', Transportation Research Part C: Emerging Technologies. Elsevier Ltd, 18(5), pp. 668-679. doi: 10.1016/j.trc.2009.08.005.

Figliozzi, M. A. (2010) 'The impacts of congestion on commercial vehicle tour characteristics and costs', Transportation Research Part E: Logistics and Transportation Review. Elsevier Ltd, 46(4), pp. 496-506. doi: 10.1016/j.tre.2009.04.005.

Gammelgaard, B. (2015) 'The emergence of city logistics: the case of Copenhagen's Citylogistik-kbh', International Journal of Physical Distribution \& Logistics Management, 45(4), pp. 333-351. doi: http:// dx.doi.org/10.1108/IJPDLM-12-2014-0291.

Gonzalez-Feliu, J. y Morana, J. (2014) 'Assessing Urban Logistics Pooling Sustainability via a Hierarchic Dashboard from a Group Decision Perspective', in Sustainable Logistics, pp. 113-135. doi: 10.1108/ S2044-994120140000006004.

Gonzalez-Feliu, J. and Muñuzuri, J. (2013) 'Decision - making tools and procedures for City Logistics', European Transport, (54), pp. 1-3.

Guedria, M., Malhene, N. y Deschamps, J.-C. (2016) 'Urban Freight Transport: From Optimized Routes to 
Robust Routes', Transportation Research Procedia. Elsevier B.V., 12(June 2015), pp. 413-424. doi: 10.1016/j.trpro.2016.02.076.

Guerlain, C., Cortina, S. y Renault, S. (2016) 'Towards a Collaborative Geographical Information System to Support Collective Decision Making for Urban Logistics Initiative', Transportation Research Procedia. Elsevier, 12, pp. 634-643. doi: 10.1016/j.trpro.2016.02.017.

Hemmelmayr, V. C., Cordeau, J.-F. y Crainic, T. G. (2012) 'An adaptive large neighborhood search heuristic for Two-Echelon Vehicle Routing Problems arising in city logistics', Computers \& Operations Research, 39(12), pp. 3215-3228. doi: 10.1016/j. cor.2012.04.007.

Holguín-Veras, J. y Sánchez-Díaz, I. (2016) 'Freight Demand Management and the Potential of ReceiverLed Consolidation programs', Transportation Research Part A: Policy and Practice, 84, pp. 109-130. doi: 10.1016/j.tra.2015.06.013.

Kim, G., Ong, Y. S., Heng, C. K., Tan, P. S. and Zhang, N. A. (2015) 'City Vehicle Routing Problem (City VRP): A Review', IEEE Transactions on Intelligent Transportation Systems, 16(4), pp. 1654-1666. doi: 10.1109/ TITS.2015.2395536.

Koç, Ç., Bektaş, T., Jabali, O. y Laporte, G. (2016) 'The impact of depot location, fleet composition and routing on emissions in city logistics', Transportation Research Part B: Methodological, 84, pp. 81-102. doi: 10.1016/j.trb.2015.12.010.

Kuo, R. J., Wibowo, B. S. y Zulvia, F. E. (2016) 'Application of a fuzzy ant colony system to solve the dynamic vehicle routing problem with uncertain service time', Applied Mathematical Modelling. Elsevier Inc., 0, pp. 1-12. doi: 10.1016/j.apm.2016.06.025.

Li, J. Q., Mirchandani, P. B. y Borenstein, D. (2009) 'A Lagrangian heuristic for the real-time vehicle rescheduling problem', Transportation Research Part E: Logistics and Transportation Review. Elsevier Ltd, 45(3), pp. 419-433. doi: 10.1016/j.tre.2008.09.002.

Li, R., Qi, M., Cheng, C. y Lai, W. (2016) 'Design of Dynamic Vehicle Routing System Based on Online Map Service', in The 13th International Conference on Service Systems and Service Management. Kunming, China: IEEE, pp. 1-5. doi: 10.1109/ICSSSM.2016.7538425.

Lin, C. (2011) 'A vehicle routing problem with pickup and delivery time windows, and coordination of transportable resources', Computers and Operations Research. Elsevier, 38(11), pp. 1596-1609. doi: 10.1016/j.cor.2011.01.021.

Lindawati, van S. J., Gohabc, M. y de Souza, R. (2014) 'Collaboration in urban logistics: motivations and barriers', International Journal of Urban Sciences, 18(2), pp. 278-290.

Lindholm, M. y Behrends, S. (2012) 'Challenges in urban freight transport planning - a review in the Baltic Sea Region', Journal of Transport Geography, 22, pp. 129-136. doi: 10.1016/j.jtrangeo.2012.01.001.

Liu, R., Xie, X., Augusto, V. y Rodriguez, C. (2013) 'Heuristic algorithms for a vehicle routing problem with simultaneous delivery and pickup and time windows in home health care', European Journal of Operational Research, 230, pp. 475-486. doi: 10.1016/j. ejor.2013.04.044.

Makhloufi, R., Cattaruzza, D., Meunier, F., Absi, N. y Feillet, D. (2015) 'Simulation of Mutualized Urban Logistics Systems with Real-time Management', Transportation Research Procedia. Elsevier, 6, pp. 365-376. doi: 10.1016/j.trpro.2015.03.027.

Malhene, N., Trentini, A., Marques, G. y Burlat, P. (2012) 'Freight consolidation centers for urban logistics solutions: The key role of interoperability', IEEE International Conference on Digital Ecosystems and Technologies. doi: 10.1109/DEST.2012.6227939.

Mańdziuk, J. y Żychowski, A. (2016) 'A memetic approach to vehicle routing problem with dynamic requests', Applied Soft Computing, 48, pp. 522-534. doi: 10.1016/j.asoc.2016.06.032.

Melo, S. M. de B. M. (2010) Evaluation of urban goods distribution initiatives towards mobility and sustainability: indicators, stakeholders and assessment tools. Univeridade do Porto. Available at: https://repositorio-aberto.up.pt/bitstream/10216/59846/1/000140073.pdf.

Montoya-Torres, J. R., Muñoz-Villamizar, A. F. y Vega-Mejía, C. A. (2016) 'On the impact of collaborative strategies for goods delivery in city logistics', Production Planning \& Control: The Management of Operations, 27(6), pp. 443-455.

Muñoz-Villamizar, A. F., Montoya-Torres, J. R., Angel, J. A. y Cáceres-Cruz, J. (2013) 'A simulation-based algorithm for the integrated location and routing problem in urban logistics', in Pasupathy, R., Kim, S.-H., Tolk, A., Hill, R., and Kuhl, M. E. (eds) 2013 
Winter simulation conference, pp. 2032-2041. doi: 10.1109/WSC.2013.6721581.

Muñoz-Villamizar, A. F., Montoya-Torres, J. R. y Vega-Mejía, C. A. (2015) 'Non-collaborative versus collaborative last-mile delivery in urban systems with stochastic demands', in 7th Industrial Product-Service Systems Conference - PSS, industry transformation for sustainability and business. Elsevier, pp. 263-268. doi: 10.1016/j.procir.2015.02.147.

Muñuzuri, J. (2003) La logística urbana de mercancías: Soluciones, modelado y evaluación. Universidad de Sevilla.

Muñuzuri, J., Cortés, P., Guadix, J. y Onieva, L. (2012) 'City logistics in Spain: Why it might never work', Cities, 29(2), pp. 133-141. doi: 10.1016/j.cities.2011.03.004.

Muñuzuri, J., Cortés, P., Onieva, L. y Guadix, J. (2009) 'Modeling freight delivery flows: Missing link of urban transport analysis', Journal of urban planning and development, 135(3), pp. 91-99.

Muñuzuri, J., Cortés, P., Onieva, L. y Guadix, J. (2012) 'Estimation of daily vehicle flows for urban freight deliveries', Journal of urban planning and development, 128(1), pp. 43-52.

Nguyen, V. P., Prins, C. y Prodhon, C. (2012) 'Solving the two-echelon location routing problem by a GRASP reinforced by a learning process and path relinking', European Journal of Operational Research. Elsevier B.V., 216(1), pp. 113-126. doi: 10.1016/j. ejor.2011.07.030

Nuzzolo, A. y Comi, A. (2014) 'Urban freight demand forecasting: A mixed quantity/delivery/vehicle-based model', Transportation Research Part E: Logistics and Transportation Review, 65, pp. 84-98. doi: 10.1016/j.tre.2013.12.014.

Palacio-León, 0. y Adarme Jaimes, W. (2014) ‘Coordinación de inventarios: Un caso de estudio para la logística de ciudad', DYNA, 81(186), pp. 295-303.

Pillac, V., Gendreau, M., Guéret, C. y Medaglia, A. L. (2013) 'A review of dynamic vehicle routing problems', European Journal of Operational Research. Elsevier B.V., 225(1), pp. 1-11. doi: 10.1016/j.ejor.2012.08.015.

Quak, H. . y De Koster, M. B. . (2009) 'Delivering goods in urban areas: how to deal with urban policy resctrictions and the enviroment.pdf', Transportation Science, 43(2), pp. 211-227.
Qureshi, A. G., Taniguchi, E. y Yamada, T. (2009) 'An exact solution approach for vehicle routing and scheduling problems with soft time windows', Transportation Research Part E: Logistics and Transportation Review, 45(6), pp. 960-977. doi: 10.1016/j. tre.2009.04.007.

Qureshi, A. G., Taniguchi, E. y Yamada, T. (2012) 'A Microsimulation Based Analysis of Exact Solution of Dynamic Vehicle Routing with Soft Time Windows', Procedia - Social and Behavioral Sciences, 39, pp. 205-216. doi: 10.1016/j.sbspro.2012.03.102.

Russo, F. y Comi, A. (2011) 'A model system for the ex-ante assessment of city logistics measures', Research in Transportation Economics. Elsevier Ltd, 31(1), pp. 81-87. doi: 10.1016/j.retrec.2010.11.011.

Sanz, G., Pastor, R. y Benedito, E. (2013) 'Distribución urbana de mercancías: Descripción y clasificación de soluciones existentes e implementación de dos soluciones novedosas', DYNA, 80(179), pp. 6-13. Available at: http://www. scopus.com/inward/record.url?eid=2-s2.0 84879151133\&partnerID=tZOtx3y1.

Schliwa, G., Armitage, R., Aziz, S., Evans, J. y Rhoades, J. (2015) 'Sustainable city logistics — Making cargo cycles viable for urban freight transport', Research in Transportation Business \& Management, 15, pp. 50-57. doi: 10.1016/j.rtbm.2015.02.001.

Sun, S. C., Duan, Z. Y. y Chen, C. (2014) 'Freight Transport Demand Forecasting in Urban Logistics Planning: A Case Study of Yiwu City', Applied Mechanics and Materials, 505-506, pp. 915-921. doi: 10.4028/www. scientific.net/AMM.505-506.915.

Tadić, S., Zečević, S. y Krstić, M. (2014) 'Ranking of Logistics System Scenarios for Central Business District', Promet-Traffic \& Transportation, 26(2), pp. 159167. doi: $10.7307 /$ ptt.v26i2.1349.

Tadić, S., Zečević, S. y Krstić, M. (2015) 'City Logistics Status and Trends', International Journal for Traffic and Transport Engineering, 5(3), pp. 319-343. doi: http://dx.doi.org/10.7708/ijtte.2015.5(3).09.

Taniguchi, E. (2014) 'Concepts of City Logistics for Sustainable and Liveable Cities', Procedia - Social and Behavioral Sciences. Elsevier, 151, pp. 310-317. doi: 10.1016/j.sbspro.2014.10.029.

Taniguchi, E. (2015) 'City Logistics for Sustainable and Liveable Cities', in Green Logistics and Transportation, pp. 49-60. doi: 10.1007/978-3-319-17181-4_4. 
Taniguchi, E., Thompson, R. G. y Yamada, T. (2014) 'Recent Trends and Innovations in Modelling City Logistics', Procedia - Social and Behavioral Sciences. Elsevier B.V., 125, pp. 4-14. doi: 10.1016/j.sbspro.2014.01.1451.

Taniguchi, E., Thompson, R. G. y Yamada, T. (2016) 'New Opportunities and Challenges for City Logistics', Transportation Research Procedia. Elsevier B.V., 12(June 2015), pp. 5-13. doi: 10.1016/j.trpro.2016.02.004.

Taniguchi, E., Thompson, R. G., Yamada, T. y Van Duin, J. H. . (2001) City logistics: Network modelling and intelligent transport systems. Elsevier Science.

United Nations Department of Economic and Social Affairs (2014) World Urbanization Prospects, World Urbanization Prospects. 2014 Revision. Highlights. doi: 10.4054/DemRes.2005.12.9.

Woudsma, C., Jensen, J. F., Kanaroglou, P. y Maoh, H. (2008) 'Logistics land use and the city: A spatial-temporal modeling approach', Transportation Research Part E: Logistics and Transportation Review, 44(2), pp. 277-297. doi: 10.1016/j.tre.2007.07.006.

Yan, S., Lin, J. R. y Lai, C. W. (2013) 'The planning and realtime adjustment of courier routing and scheduling under stochastic travel times and demands', Transportation Research Part E: Logistics and Transportation Review. Elsevier Ltd, 53(1), pp. 34-47. doi: 10.1016/j.tre.2013.01.011.

Yang, Z., van Osta, J.-P., van Veen, B., van Krevelen, R., van Klaveren, R., Stam, A., Kok, J., Bäck, T. y Emmerich, M. (2016) 'Dynamic vehicle routing with time windows in theory and practice', Natural Computing. doi: 10.1007/s11047-016-9550-9.

Zhang, J., Lam, W. H. K. y Chen, B. Y. (2016) 'On-time delivery probabilistic models for the vehicle routing problem with stochastic demands and time windows', European Journal of Operational Research. Elsevier Ltd., 249(1), pp. 144-154. doi: 10.1016/j. ejor.2015.08.050.

Zhu, L., Rousseau, L. M., Rei, W. y Li, B. (2014) 'Paired cooperative reoptimization strategy for the vehicle routing problem with stochastic demands', Computers and Operations Research, 50, pp. 1-13. doi: 10.1016/j.cor.2014.03.027.

Zufferey, N., Cho, B. Y. y Glardon, R. (2016) 'Dynamic Multi-trip Vehicle Routing with Unusual Timewindows for the Pick-up of Blood Samples and De- livery of Medical Material', Proceedings of 5th the International Conference on Operations Research and Enterprise Systems, (Icores), pp. 366-372. doi: $10.5220 / 0005733303660372$

\section{PARA CITAR ESTE ARTÍCULO / TO REFERENCE THIS ARTICLE / PARA CITAR ESTE ARTIGO /}

Arango Serna, M.D.; Gómez Marín, C.G.; Serna Urán, C.A. (2017). Modelos logísticos aplicados en la distribución urbana de mercancías. Revista EIA, 14(28), julio-diciembre, pp. 57-76. [Online]. Disponible en: https://doi.org/10.24050/ reia.v14i28.1055 